\title{
Mycobacterium nonchromogenicum en un paciente con neumoconiosis: reporte de un caso en atención primaria
}

Mycobacterium nonchromogenicum in a Patient with Pneumoconiosis: Report of a Case in Primary Care

\author{
Alejandra Melisa Villadiego ${ }^{a}$ \\ Cortina \\ Pontificia Universidad Javeriana, Colombia \\ ERnesto Pardo Rubio \\ Javesalud IPS, Colombia
}

a Correspondencia: alejavilla@gmail.com

\section{RESUMEN}

La neumoconiosis es una patología pulmonar crónica producida por el daño tisular que originan algunos tóxicos al ser inhalados durante ciertos periodos. El caso presentado corresponde a un paciente inmunocompetente con silicoantracosis, un tipo de neumoconiosis, en quien se detecta infección pulmonar por Mycobacterium nonchromogenicum en un centro de atención primaria en Bogotá, Colombia, poco común y de gran interés médico.

Palabras clave

micobacterias no tuberculosas; neumoconiosis; atención primaria de salud.

\begin{abstract}
Pneumoconiosis is a chronic lung disease, caused by the inhalation of toxins that produces lung tissue damage; one type of pneumoconiosis is silicoanthracosis. In this reported case, we described an immunocompetent patient that has a pulmonary infection by Mycobacterium nonchromogenicum at a primary care center in Bogotá, Colombia; being an unusual case.

Keywords
\end{abstract}

Mycobacterium nontuberculous; pneumoconiosis; primary health care.

\section{Introducción}

La neumoconiosis es una enfermedad pulmonar crónica en la cual existe daño pulmonar del tejido intersticial, ocasionado por la exposición inhalada a agentes tóxicos. La silicoantracosis es un tipo de neumoconiosis, causada por la sílice y el carbón (1), lo cual constituye un problema de salud pública mundial y principalmente en países del tercer mundo, ya que es típica en trabajadores de minería. Además, aparte de que ocasiona altos costos al sistema de salud y

Cómo citar: Villadiego Cortina AM, Pardo Rubio E. Mycobacterium nonchromogenicum en un paciente con neumoconiosis: reporte de un caso en atención primaria. Univ. Med. 2018;59(4). doi: https:// doi.org/10.11144/Javeriana.umed59-4.myco 
produce discapacidad funcional, precisa medicamentos de uso prolongado y altas tasas de hospitalización (1).

Las micobacterias son microrganismos pertenecientes a la familia de bacterias Mycobacteriaceae, son bacilos aerobios grampositivos no esporulados con un tamaño aproximado de 5 micras. En este grupo se encuentra la Mycobacterium tuberculosis y leprae, también conocidas como típicas. Son ácidoalcohol resistentes, inmóviles y de crecimiento lento (2). La prevalencia de infección en la población general no sobrepasa el $1 \%$ y aumenta al 5\% en la población con VIH-sida (3).

El Mycobacterium nonchromogenicum es un tipo de micobacteria considerada atípica, que produce infección en diferentes órganos y sistemas, principalmente en pacientes con alguna alteración en su sistema inmunológico; por esto se considera una infección de tipo oportunista. Se describen algunos reportes de infección en pacientes con neumopatías crónicas como neumoconiosis, enfermedad pulmonar obstructiva crónica, entre otras.

$\mathrm{Su}$ cuadro clínico se caracteriza por la aparición de síntomas respiratorios como disnea, tos y expectoración, pérdida de peso y dolor torácico. Este caso es de gran importancia médica, ya que en la literatura existente son escasos los datos epidemiológicos reportados en Latinoamérica y favorece la sospecha diagnóstica hacia este tipo de microrganismos atípicos dentro de la consulta externa en pacientes inmunocompetentes.

\section{Caso clínico}

El caso corresponde a un hombre de 58 años de edad, quien consultó por agudización de disnea en los últimos 4 meses, al pasar de $1 / 4$ a $3 / 4$ según la Escala Modificada de la Disnea. El paciente tenía antecedente de silicoantracosis pulmonar, diagnosticada desde 2009 mediante biopsia, debido a la exposición a diferentes polvos utilizados en construcción durante 30 años. Había sido manejada con oxigenoterapia $12 \mathrm{~h}$ al día y era atendido en el programa de enfermedades crónicas en un centro de salud de atención primaria.

Durante la consulta, se obtuvieron los siguientes signos vitales y hallazgos en el examen físico: 78 latidos por minuto, 18 respiraciones por minuto, $37{ }^{\circ} \mathrm{C}$ de temperatura, saturación de oxígeno del $88 \%$, peso de $65 \mathrm{~kg}$, índice de masa corporal de 24. En la auscultación cardiopulmonar se evidenciaron roncus diseminados en ambos campos pulmonares como único hallazgo positivo.

Se solicitó la realización de una baciloscopia seriada de esputo, que fue negativa, y posterior cultivo. La radiografía de tórax evidenció la presencia de nódulos pulmonares e infiltrados intersticiales micronodulares, compatibles con silicoantracosis (figura 1). También se le realizó perfil hepático y hemograma, que estaban dentro de la normalidad. En el momento de la consulta, se realizó manejo con broncodilatadores.

\section{Figura 1}

Radiografía de tórax del paciente, proyección posteroanterior que evidencia infiltrados micronodulares parabronquiales y signos de hipertensión pulmonar, entre otros hallazgos como atelectasia del lóbulo inferior derecho y elongación de la aorta

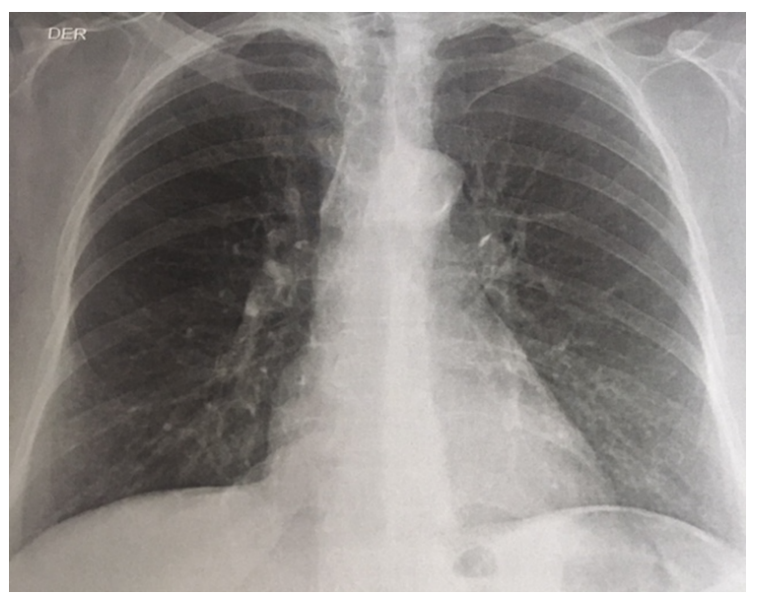

El cultivo fue positivo para Mycobacterium tuberculosis a los 3 meses de su recolección; Después de este resultado, se realizó la consulta con el servicio de neumología, que inició la primera fase de tratamiento para tuberculosis 
pulmonar, previa notificación epidemiológica, con isoniacida de $75 \mathrm{mg}$, rifampicina de $150 \mathrm{mg}$, pirazinamida de $400 \mathrm{mg}$, etambutol de $275 \mathrm{mg}$ durante 6 días a la semana, durante 3 meses, y se le realizó la prueba de ELISA VIH, la cual fue negativa. En la consulta, el paciente refirió persistencia de la disnea, a pesar del tratamiento inicial.

A los 3 meses de iniciado el tratamiento antibiótico, se obtuvo la tipificación del germen obtenido en el cultivo y se evidenció el crecimiento de Mycobacterium nonchromogenicum. En la consulta de control, posterior a la primera fase de tratamiento, el paciente refirió mejoría de la disnea de $3 / 4$ a $1 / 4$, según la Escala Modificada de la Disnea, al volver al valor inicial previo al cuadro de agudización. Siguiente a eso, se le realizó un cultivo de control y se inició la segunda fase del tratamiento, ya que había una respuesta clínica satisfactoria, con isoniacida de $150 \mathrm{mg}$ y rifampicina de $150 \mathrm{mg}$, por 3 días a la semana durante 3 meses. Finalmente, se obtuvo un reporte negativo para micobacterias en el último cultivo realizado.

Actualmente, el paciente se encuentra estable, sin agudización de sus síntomas respiratorios y continúa en controles con neumología cada 3 meses con solicitud de baciloscopia de control anual.

\section{Discusión}

En Colombia existen programas de atención para patologías crónicas pulmonares como en Javesalud IPS, un centro de atención primaria, que cuenta con un programa para enfermedad pulmonar obstructiva crónica y manejo integral de patologías crónicas. En este artículo se informa el caso de un paciente con una infección por Mycobacterium nonchromogenicum diagnosticado en esta institución, siendo el primer caso detectado.

En la literatura sobre el tema hay algunos estudios de salud ocupacional sobre silicoantracosis realizados en minas de Colombia como en Cerrejón, La Guajira, con el fin de minimizar el riesgo y las consecuencias de la exposición a agentes tóxicos (4). Según la Organización Mundial de la Salud, su prevalencia está alrededor del $42 \%$ en personas expuestas, lo que representa un promedio de 1.240 .000 años de vida saludables perdidos y costos significativos para el sistema de salud de cada país, teniendo una tasa de mortalidad en Estados Unidos de 13,2 por millón en 2000 (1). El diagnóstico parte de la observación de hallazgos radiológicos sugestivos, como lesiones nodulares o infiltrados pulmonares intersticiales; pero su diagnóstico definitivo es mediante biopsia pulmonar, como se evidenció en este caso. El tratamiento consiste en manejo con inhaloterapia y oxigenoterapia. Como principal complicación se presenta el cor pulmonale, la falla ventilatoria severa y la muerte (5).

En cuanto a la infección por Mycobacterium nonchromogenicum, en las imágenes diagnósticas se evidencia un nódulo que suele ser único, generalmente ubicado en los lóbulos superiores, y al realizar un cultivo en esputo, se evidencia el microrganismo (6).

$\mathrm{Su}$ tratamiento se centra en la antibioticoterapia, en la cual se utilizan medicamentos como el etambutol o la claritromicina. Si no se trata de modo oportuno y adecuado, puede ocasionar una sepsis (infección generalizada) o incluso la muerte (7).

El aquí presentado es un caso típico de silicoantracosis: un hombre con antecedente exposicional a trabajos industriales, a quien se le realizó dentro de sus exámenes paraclínicos cultivo de esputo que evidenció la presencia de Mycobacterium nonchromogenicum. Su tratamiento se basó en el uso de etambutol, junto a los otros medicamentos antituberculosos, ya que la tipificación del germen se realizó a los 3 meses del inicio del tratamiento; sin embargo, se obtuvo una remisión de los síntomas respiratorios y negativización del cultivo.

En la literatura sobre el tema se ha informado infección por micobacterias en un $25 \%$ de pacientes con silicoantracosis; además, se ha evidenciado una alta prevalencia de neumopatía crónica, principalmente de los tipos tuberculosis, kansasii y avium intracelulare (8); cerca de la mitad se produce por M. tuberculosis (9). Nuestro paciente, durante su toda su historia 
clínica, presentó un episodio de infección por Mycobacterium nonchromogenicum, y su único síntoma fue el deterioro de su clase funcional. No tenía factores de riesgo adicionales a su neumopatía crónica.

Es incierto que exista una susceptibilidad de padecer infecciones por micobacterias en pacientes con silicoantracosis, pero en la literatura se ha sugerido que los bacilos se pueden alojar en los nódulos silicóticos, lo que facilita su reproducción y diseminación; además, existe una alteración en la respuesta inmunológica en este tipo de pacientes (10).

La Mycobacterium nonchromogenicum, al ser una micobacteria atípica, es de rara aparición en pacientes inmunocompetentes. En este caso, llama la atención que el paciente no padece afectación inmune aparente que predisponga a este tipo de infecciones.

Adicionalmente, esta infección se considera de baja ocurrencia en pacientes con neumopatía crónica; pero no hay datos epidemiológicos específicos, pues la poca evidencia está supeditada a escasos reportes de casos que dan cuenta de la infección de este microrganismo en otros órganos y sistemas (meninges y cavidad sinovial) (6). Este el primer paciente con infección por Mycobacterium nonchromogenicum detectado en esta institución.

Este caso es ilustrativo de cómo la atención primaria en salud es fundamental para detectar patologías atípicas en pacientes inmunocompetentes, cuando muchas veces no se sospechan. Adicionalmente, permite tratarlas en el ámbito de la consulta externa, evitando remisiones a altos niveles de complejidad, y realizarles seguimiento a estos casos que se producen con muy baja frecuencia; pero que se deben tener en cuenta especialmente en pacientes con patologías crónicas a quienes se les realiza un seguimiento periódico.

\section{Referencias}

1. Briceño L, Romero M. Prevalencia de neumoconiosis y hallazgos espirométricos en trabajadores expuestos a polvo de carbón en minería subterránea en el departamento de Cundinamarca, Colombia, 2014 [internet]. Disponible en: http://repository.urosario.edu.co/h andle/10336/11804

2. Surco Luna V, Gavincha Quispe CV. Micobacterias. Rev Act Clin Med [internet]. 2014;49. Disponible en: htt p://www.revistasbolivianas.org.bo/sciel o.php?pid=S2304-376820140010000 $07 \&$ script $=$ sci_arttext

3. Alvarado F, Bustillo JG, Divels E. Prevalencia de micobacterias en pacientes $\mathrm{VIH}$ SIDA. EnColombia [internet]; 2008. Disponible: https://encolombia.com/m edicina/revistas-medicas/neumologia/v ns-134/neum134-01prevalencia/

4. Ramírez E, Herrera F, Zúñiga I et al. Silicoantracosis en El Cerrejón: propuesta para la acción. Car Med AISBol. 2002;17(1):17-21.

5. Polo B. Guías de neumoconiosis. Bogotá: Ministerio de la Protección Social y Pontificia Universidad Javeriana;2007.

6. Sawai T, Inoue Y, Doi S, Izumikawa $\mathrm{K}$, Ohno $\mathrm{H}$, Yanagihara K, et al. A case of mycobacterium nonchromogenicum pulmonary infection showing multiple nodular shadows in an inmunocompetent patient. Diagn Microbiol Infect Dis. 200654(4):311-4.

7. Kekkaku J, Wallace RJ Jr, Kilburn JO, Butler WR, Warren NG, Tsukamura M. Chronic tenosynovitis of the hand due to mycobacterium nonchromogenicum: Use of high performance liquid chromatography for identification of isolates. Rev Infect Dis. 1991;13(5):857-64.

8. Tsukamura M, Kita N, Otsuka W, Shimoide H. A study of the taxonomy of the mycobacterium nonchromogenicum 
complex and report of six cases of lung infection due to mycobacterium nonchromogenicum. Microbiol Immunol. 198327 (3):219-36.

9. Greenberg MI, Waksman J, Curtis J. Silicosis: A review. Dis Mon. 2007;53(8):394-416.

10. Hnizdo E, Murray J. Risk of Pulmonary tuberculosis relative to silicosis and exposure to silica dust in south African gold miners. Occup Environ Med. 1998;55(7):496-502. 